TRANSACTIONS OF THE

AMERICAN MATHEMATICAL SOCIETY

Volume 351, Number 1, January 1999, Pages 193-210

S 0002-9947(99)02289-8

\title{
REDUCIBILITY OF SOME INDUCED REPRESENTATIONS OF $p$-ADIC UNITARY GROUPS
}

\author{
FIONA MURNAGHAN AND JOE REPKA
}

\begin{abstract}
In this paper we study reducibility of those representations of quasi-split unitary $p$-adic groups which are parabolically induced from supercuspidal representations of general linear groups. For a supercuspidal representation associated via Howe's construction to an admissible character, we show that in many cases a criterion of Goldberg for reducibility of the induced representation reduces to a simple condition on the admissible character.
\end{abstract}

\section{INTRODUCTION}

Let $K$ be a quadratic extension of a $p$-adic field $F$ of characteristic zero and odd residue characteristic. Let $G^{\prime}$ and $G^{\prime \prime}$ be the $F$-rational points of the quasi-split unitary groups in $2 n$ and $2 n+1$ variables, respectively, defined with respect to the extension $K / F$. Let $G=G L_{n}(K)$. Denote the kernel of the norm map from $K^{\times}$ to $F^{\times}$by $K^{1}$. The group $G^{\prime}$, resp. $G^{\prime \prime}$, has a maximal parabolic subgroup $P^{\prime}$, resp. $P^{\prime \prime}$, with Levi factor isomorphic to $G$, resp. $G \times K^{1}$. Let $\pi$ be an irreducible unitary supercuspidal representation of $G$, and $\xi$ a character of $K^{1}$. Define a supercuspidal representation of $\Pi_{\xi}$ of $G \times K^{1}$ by $\Pi_{\xi}(x, \alpha)=\pi(x) \xi\left(\operatorname{det}_{0}(x \eta(x)) \alpha\right)$, for $x$ in $G$ and $\alpha \in K^{1}$. Here $\operatorname{det}_{0}$ is the determinant on $G$, and $\eta$ is the automorphism of $G$ taking $x$ to ${ }^{t} \bar{x}^{-1}$, where the bar denotes the usual action of the non-trivial element of $\operatorname{Gal}(K / F)$ on matrices with entries in $K$. Set

$$
I(\pi)=\operatorname{Ind}_{P^{\prime}}^{G^{\prime}}(\pi \otimes 1)
$$

and

$$
I\left(\Pi_{\xi}\right)=\operatorname{Ind}_{P^{\prime \prime}}^{G^{\prime \prime}}\left(\Pi_{\xi} \otimes 1\right) .
$$

As it is a necessary condition for reducibilty of $I(\pi)$, and also for $I\left(\Pi_{\xi}\right)$, we assume that $\pi$ is equivalent to $\pi \circ \eta$. In [G2], Goldberg proves that, under this assumption, $I(\pi)$ is reducible, resp. $I\left(\Pi_{\xi}\right)$ is irreducible, if and only if the sum of two particular $\eta$-twisted orbital integrals vanishes for every choice of matrix coefficient of $\pi$.

Suppose that $\pi$ arises via the construction of Howe $([\mathrm{H}])$ from an admissible character $\theta$ of the multiplicative group of a tamely ramified degree $n$ extension $E$ of $K$. We show that $\pi$ is equivalent to $\pi \circ \eta$ if and only $\theta \circ \sigma=\theta^{-1}$ for some involutive automorphism of $E / F$ which is non-trivial on $K$. In this paper, we prove that, for many such $\pi$, Goldberg's reducibility criterion reduces to a simple condition on $\theta$. If $L$ is the fixed field of $\sigma$, then either $\theta \mid L^{\times}$is trivial or is equal to the quadratic

Received by the editors November 14, 1996.

1991 Mathematics Subject Classification. Primary 22E50.

Research supported in part by NSERC.

(C)1999 American Mathematical Society 
character of $L^{\times}$associated to $E / L$ by class field theory. When $E$ is ramified over $L$ and $\theta \mid L^{\times}$is trivial, we show that the sum of $\eta$-twisted orbital integrals which appears in the reducibility criterion is non-zero for a particular choice of matrix coefficient of $\pi$. When $E$ is unramified over $L$, we get a similar result under some additional assumptions on $\theta$. In an earlier paper ([MR]), using a reducibility criterion of Shahidi ([Sh]), we obtained the same type of results for representations of split classical groups induced from self-contragredient supercuspidal representations of general linear groups. Many of the results of this paper are proved by modifying proofs of analogous results of $[\mathrm{MR}]$.

In $\S 2$, we derive the relation between the equivalence of $\pi$ and $\pi \circ \eta$ and existence of $\sigma$ as above. In particular, it follows from a result of Adler ([A]) that existence of such an involution $\sigma$ guarantees existence of such supercuspidal representations $\pi$. We also discuss properties of the Howe factorization of $\theta$ relative to $\sigma$.

The $\eta$-twisted orbital integrals in Goldberg's criterion can be expressed as integrals over certain sets of fixed points in $G$ of an involutive anti-automorphism $\varphi$ of $\mathfrak{g} l_{n}(K)$. The third section contains a description of the action of $\varphi$ on filtrations of the parahoric subalgebra attached to the extension $E / K$, and on related subgroups of $G$.

The representation $\pi$ is induced from an irreducible representation $\kappa$ of an open compact subgroup $H_{0}$ of $G$. In $\S 4$, we state the reducibility criterion of [G2], and show that for an appropriately chosen finite sum $f_{\pi}$ of matrix coefficients of $\pi$, each of the two relevant $\eta$-twisted orbital integrals $\Phi_{\eta}\left(h_{k}, f_{\pi}\right), k=1,2$, reduces to the integral of the character of $\kappa$ over a certain $\varphi$-invariant subset of $H_{0}$.

In $\S 5$, we give some values of the character of $\kappa$, and summarize some results from [MR] relating properties of $\kappa$ and certain extensions of $F$ contained in $E$. We prove that if $\kappa$ is one-dimensional, then $\Phi_{\eta}\left(h_{k}, f_{\pi}\right)>0, k=1,2$.

Up to a character of $H_{0}$, the inducing representation $\kappa$ is a tensor product of finitely many representations $\kappa_{i}$ corresponding to the Howe factors $\theta_{i}, i=1, \ldots, r$, of the admissible character $\theta$. In $\S 6$, we show that if a Heisenberg representation is used in the construction of one of these factors, then the character $\chi_{i}$ of $\kappa_{i}$ is real-valued on the set of $\varphi$-invariant points in $H_{0}$. We then compute the value of certain signs appearing in the formula for $\chi_{i}$.

Next, in $\S 7$, we consider the case when the representation $\kappa_{r}$ is defined in terms of a cuspidal representation of a finite general linear group. Assuming that $\kappa_{i}$ is one-dimensional for $1 \leq i \leq r-1$, we outline how to modify the arguments of [MR] to express $\Phi_{\eta}\left(h_{k}, f_{\pi}\right), k=1,2$, in terms of values of $\theta$ and sums of $\chi_{r}$ over various subsets of $H_{0}$. As shown in [MR], these sums of values of $\chi_{r}$ can be expressed in terms of Deligne-Lusztig characters of non-connected finite reductive groups which were computed in [MR]. This allows us to relate the signs of $\Phi_{\eta}\left(h_{k}, f_{\pi}\right), k=1,2$, and $\theta \mid L^{\times}$.

The main results of the paper are Theorems 8.1 and 8.3. We state conditions on $\theta \mid L^{\times}$which guarantee that $\Phi_{\eta}\left(h_{k}, f_{\pi}\right)>0, k=1,2$, and hence that $I(\pi)$ is irreducible, resp. $I\left(\Pi_{\xi}\right)$ is reducible.

In analogy with the situation in [Sh], the reducibility criterion of [G2] can be interpreted in terms of the conjectural theory of twisted endoscopy ([KS1],[KS2]). For $n=2$ and 3, this is discussed in [G1] and in $\S 4$ of [G2], respectively. Under the conditions on $\theta$ given in $\S 8$ of this paper, the representation $\pi$ should be a lift from the unitary group in $n$ variables (see $\S \S 4,6$ of [G2]). 


\section{Howe FaCTORIZATIONS OF ADMISSIBLE CHARACTERS}

Let $F$ be a $p$-adic field of characteristic zero and odd residual characteristic. If $F^{\prime}$ is a finite extension of $F$, we will use the notation $\mathcal{O}_{F^{\prime}}, \mathfrak{p}_{F^{\prime}}$, and $\varpi_{F^{\prime}}$ for the ring of integers in $F^{\prime}$, maximal ideal in the ring of integers, and a uniformizer in $F^{\prime}$, respectively. The norm and trace maps from $F^{\prime}$ to $F$ will be denoted by $N_{F^{\prime} / F}$ and $\operatorname{tr}_{F^{\prime} / F}$, respectively. Fix a quadratic extension $K$ of $F$. For $n \geq 2$, set $G=G L_{n}(K)$; we let $x \mapsto \bar{x}$ denote the action of the non-trivial element of the Galois group of $K / F$ on $G$ (apply the automorphism to matrix entries). Set $\eta(x)={ }^{t} \bar{x}^{-1}$. Let $\pi$ be an irreducible supercuspidal representation of $G$ such that $\pi \circ \eta$ is equivalent to $\pi$ (denoted by $\pi \circ \eta \sim \pi$ ). Now suppose that $\pi$ arises via Howe's construction from an admissible character $\theta$ of $E^{\times}$, where $E / K$ is tamely ramified of degree $n$. Note that $E / F$ may not be Galois; we use the notation $\operatorname{Aut}(E / F)$ to refer to the set of automorphisms of $E$ that fix $F$ pointwise, and similarly for $\operatorname{Aut}(E / K)$. Note that $\theta$ is admissible over $K$, but might not be admissible over $F$. Assume that $\pi$ (hence $\theta$ ) is unitary. The above condition on $\pi$ translates into a condition on $\theta$.

Lemma 2.1. $\pi \sim \pi \circ \eta$ if and only if there exists an involution $\sigma \in \operatorname{Aut}(E / F)$ such that $\sigma \mid K \not \equiv i d$ and $\theta \circ \sigma=\theta^{-1}$.

Proof. $(\Rightarrow) \quad$ Take an embedding $\tau$ of $E$ into the algebraic closure of $F$ having the property that $\tau \mid K$ is the non-trivial element of $\operatorname{Gal}(K / F)$. Let $E^{\prime}=\tau(E)$. Then we can set $\theta^{\prime}(\tau(\alpha))=\theta(\alpha), \alpha \in E^{\times}$and observe that $\theta^{\prime}$ is attached to the representation $x \mapsto \pi(\bar{x})$. But we also know that $x \mapsto \pi\left({ }^{t} x^{-1}\right)$ is attached to $\theta^{-1}$. So the condition on $\pi$ forces $\theta^{\prime}$ and $\theta^{-1}$ to be conjugate (over $K$ ): there is a field isomorphism $\tau^{\prime}: E^{\prime} \rightarrow E$ which fixes $K$ pointwise such that $\theta^{-1}\left(\tau^{\prime}\left(\alpha^{\prime}\right)\right)=\theta^{\prime}\left(\alpha^{\prime}\right)$, $\alpha^{\prime} \in E^{\prime \times}$. Set $\sigma=\tau^{\prime} \circ \tau$. Then $\sigma \in \operatorname{Aut}(E / F)$. The automorphism $\sigma$ has the property that $\sigma \mid K$ is the non-trivial element of $\operatorname{Gal}(K / F)$ and also that $\theta \circ \sigma=\theta^{-1}$.

What remains is to show that $\sigma$ is an involution. Note that $\theta \circ \sigma^{2}=\theta$, and $\sigma^{2} \in \operatorname{Aut}(E / K)$. Suppose the order of $\sigma^{2}$ is $k>1$. Write $E^{\sigma^{2}}$ for the fixed field of $\sigma^{2}$. Then $\left[E: E^{\sigma^{2}}\right] \leq k$. But $1, \sigma^{2}, \sigma^{4}, \ldots, \sigma^{2(k-1)}$ are $k$ distinct automorphisms of $E$ fixing $E^{\sigma^{2}}$ pointwise. This shows that $E / E^{\sigma^{2}}$ is normal, with $\left[E: E^{\sigma^{2}}\right]=k$, and therefore Galois. Since $\theta \circ \sigma^{2}=\theta$, we find that for any $t \in E^{\times}, \theta\left(\frac{t}{\sigma^{2}(t)}\right)=1$. By Hilbert 90, this shows that $\theta$ is trivial on the elements of norm 1, so $\theta$ factors through the norm $N_{E / E^{\sigma^{2}}}$. This contradicts the admissibility of $\theta$, proving that $\sigma$ is indeed an involution.

$(\Leftarrow) \quad$ If there is an involution $\sigma$ as in the statement of the lemma, then, as above, $x \mapsto \pi(\bar{x})$ is equivalent to $x \mapsto \pi\left({ }^{t} x^{-1}\right)$, so $\pi \sim \pi \circ \eta$.

Note that in contrast to the situation in [MR], $\sigma$ acts non-trivially on the base field $K$ over which the supercuspidal representation is defined.

Lemma 2.2. Suppose $E / K$ is a tamely ramified extension of degree $n$. The following are equivalent:

(i) There exists an involution $\sigma \in \operatorname{Aut}(E / F)$ such that $\left.\sigma\right|_{K} \not \equiv i d$.

(ii) There exist irreducible unitary supercuspidal representations $\pi$ of $G$ associated by the construction of Howe to admissible characters $\theta$ of $E^{\times}$and satisfying $\pi \sim$ $\pi \circ \eta$.

Proof. Part (ii) implies (i) by Lemma 2.1. 
$(i) \Rightarrow(i i)$ : The fixed field of $\sigma$ is of index 2 in $E$. The argument given in the proof of Theorem 6.1 of $[\mathrm{A}]$ shows that there exists a character $\theta$ of $E^{\times}$that is admissible over $F$ and such that $\theta \circ \sigma=\theta^{-1}$. Admissibility over $F$ implies admissibility over $K$, and (ii) follows by Lemma 2.1 .

Assume that $\pi$ and $\theta$ are as in Lemma 2.1. The admissible character $\theta$ of $E^{\times}$ has a Howe factorization (see $[\mathrm{H}],[\mathrm{M}])$ :

$$
\theta=\left(\Lambda \circ N_{E / K}\right) \theta_{r}\left(\theta_{r-1} \circ N_{E / E_{r-1}}\right) \cdots\left(\theta_{2} \circ N_{E / E_{2}}\right)\left(\theta_{1} \circ N_{E / E_{1}}\right) .
$$

Here $\theta$ uniquely determines the tower of fields $K=E_{0} \subset E_{1} \subset \cdots \subset E_{r}=E$ and $\Lambda, \theta_{1}, \ldots, \theta_{r}$ are quasi-characters of $E_{0}^{\times}, E_{1}^{\times}, \ldots, E_{r}^{\times}$, respectively. Comparison of the Howe factorizations of $\theta$ and $\theta \circ \sigma$ shows that $\sigma\left(E_{i}\right)=E_{i}$ for each $i$, although we shall see that $\sigma$ does not fix $E_{i}$ pointwise. Each quasi-character $\theta_{i}$ is generic over $E_{i-1}([\mathrm{H}])$. The conductoral exponents are unique and satisfy

$$
f_{E}\left(\theta_{1} \circ N_{E / E_{1}}\right)>\cdots>f_{E}\left(\theta_{r}\right)>0
$$

If $f_{E}\left(\Lambda \circ N_{E / K}\right) \leq f_{E}\left(\theta_{1} \circ N_{E / E_{1}}\right)$, note that it is possible to absorb $\Lambda \circ N_{E / K}$ into $\theta_{1} \circ N_{E / E_{1}}$ and write $\left(\Lambda \circ N_{E / K}\right)\left(\theta_{1} \circ N_{E / E_{1}}\right)=\left(\theta_{1}^{\prime} \circ N_{E / E_{1}}\right)$ for a $\theta_{1}^{\prime}$ that is still generic over $E_{1}$. Because of this, we can choose $\theta_{1}$ such that either $\Lambda \equiv 1$ or $f_{E}\left(\Lambda \circ N_{E / K}\right)>f_{E}\left(\theta_{1} \circ N_{E / E_{1}}\right)$. For each $i=1, \ldots, r-1$, choose an element $c_{i} \in E_{i}$ that "represents" $\theta_{i}$ in the sense that

$$
\theta_{i}(1+x)=\psi\left(\operatorname{tr}_{E_{i} / K}\left(c_{i} x\right)\right), \quad \text { for } \quad x \in \mathfrak{p}_{E_{i}}^{\left[\frac{f_{E_{i}}\left(\theta_{i}\right)+1}{2}\right]},
$$

where $\psi=\psi_{0} \circ \operatorname{tr}_{K / F}$ and $\psi_{0}$ is a character of the additive group $F$ with conductor $\mathfrak{p}_{F}$; we must have $c_{i} \in \mathfrak{p}_{E_{i}}^{-f_{E_{i}}\left(\theta_{i}\right)+1} \backslash \mathfrak{p}_{E_{i}}^{-f_{E_{i}}\left(\theta_{i}\right)+2}$ (see $[\mathrm{H}],[\mathrm{M}]$ ). Note that the genericity of $\theta_{i}$ implies that $c_{i}$ generates $E_{i}$ over $E_{i-1}$. If $i=r$ and $f_{E}\left(\theta_{r}\right)>1$, choose $c_{r}$ as above.

Let $\sigma$ be as in Lemma 2.1.

Lemma 2.3. The characters $\Lambda$ and $\theta_{i}$, and the elements $c_{i}$ can be chosen so that

(i) $\Lambda, \theta_{i}$ are unitary,

(ii) $\Lambda \circ N_{E / K} \circ \sigma=\left(\Lambda \circ N_{E / K}\right)^{-1}, \quad \theta_{i} \circ N_{E / E_{i}} \circ \sigma=\left(\theta_{i} \circ N_{E / E_{i}}\right)^{-1}$,

(iii) $\sigma\left(c_{i}\right)=-c_{i}$, if $f_{E}\left(\theta_{i}\right)>1$.

Proof. The proof is the same as the proof of Lemma 2.5 in $[\mathrm{MR}]$, noting that the adjustments made in that proof to the various characters do not affect whether or not $f_{E}\left(\Lambda \circ N_{E / K}\right)>f_{E}\left(\theta_{1} \circ N_{E / E_{1}}\right)$ (and hence whether or not $\Lambda \equiv 1$ ).

From now on we assume that $\Lambda, \theta_{i}$ and $c_{i}$ are as in Lemma 2.3.

\section{Filtrations and the map $\varphi$}

Let the notation be as in $\S 2$. We will define an antimorphism $\varphi$ of $\mathfrak{g} l_{n}(K)$ whose action on $E$ is given by $\sigma$, and so that the integrals we will be discussing can be expressed in terms of integrals over certain sets of $\varphi$-invariant points in a subgroup $H_{0}$. The subgroup $H_{0}$ is the intersection with $G$ of the subgroup $H$ of $G L_{2 n}(F)$ defined in $[\mathrm{MR}]$, and the map $\varphi$ is the restriction to $G$ of the map $\varphi$ defined there, so various properties of these maps relative to intermediate extensions, filtrations, and parahoric subgroups will follow immediately from results of [MR].

Let $L$ be the fixed field of $\sigma$ in $E$. We begin by fixing embeddings $L \hookrightarrow \mathfrak{g} l_{n}(F)$ and $E \hookrightarrow \mathfrak{g} l_{2}(L) \subset \mathfrak{g} l_{2 n}(F)$ and a symmetric matrix $s \in G L_{n}(F)$ such that $w=$ 
$\left(\begin{array}{cc}0 & s \\ -s & 0\end{array}\right)$ satisfies $w^{-1} t w=\sigma(\gamma)$ for every $\gamma \in E \subset \mathfrak{g} l_{2 n}(F)$. Then, as in [MR], we define the map $\varphi: \mathfrak{g} l_{2 n}(F) \rightarrow \mathfrak{g} l_{2 n}(F)$ by

$$
\varphi(X)=w^{-1 t} X w .
$$

By Lemma 3.4 of $[\mathrm{MR}]$, there is a symmetric matrix $\mathcal{S} \in G L_{n}(F) \subset G L_{n}(K)$ such that for $X \in \mathfrak{g} l_{n}(K)$, we have

$$
\varphi(X)=\mathcal{S}^{-1 t} X \mathcal{S}
$$

where here and from now on ${ }^{t}$. refers to the transpose in $\mathfrak{g} l_{n}(K)$ and $\bar{X}$ refers to the conjugate of $X$ by $\sigma$ acting on the entries of $X$. If $E / L$ is ramified, take $a_{L}$ to be a non-square root of unity in $L$. Otherwise, let $a_{L}=\varpi_{L}$. Then let

$$
h_{1}=\mathcal{S}^{-1} \quad \text { and } \quad h_{2}=a_{L} h_{1}=a_{L} \mathcal{S}^{-1} .
$$

Note that $h_{1}$ and $h_{2}$ are hermitian as elements of $G L_{n}(K)$ relative to the action of $\sigma$ described above. Because of the choice of $a_{L}, \operatorname{det}\left(h_{1}\right)$ and $\operatorname{det}\left(h_{2}\right)=$ $N_{E / K}\left(a_{L}\right) \operatorname{det}\left(h_{1}\right)$ both belong to $F^{\times}$and, under the assumptions on $E$ and $\sigma$ (see Lemma 2.2(i)) they lie in different cosets of $N_{K / F}\left(K^{\times}\right)$. This implies that $h_{1}$ and $h_{2}$ are representatives of the two equivalence classes of hermitian matrices in $G$.

We define various subalgebras and subgroups as in $[\mathrm{MR}]$. The parahoric $\mathcal{O}_{F^{-}}$ subalgebra $\mathcal{B} \subset \mathfrak{g} l_{2 n}(F)$ attached to the embedding $E \hookrightarrow \mathfrak{g} l_{2 n}(F)$ is defined by

$$
\mathcal{B}=\left\{X \in \mathfrak{g} l_{2 n}(F) \mid X \mathfrak{p}_{E}^{k} \subset \mathfrak{p}_{E}^{k}, \text { for all } k\right\} .
$$

For any integer $j$, we also define

$$
\mathcal{B}_{j}=\left\{X \in \mathfrak{g} l_{2 n}(F) \mid X \mathfrak{p}_{E}^{k} \subset \mathfrak{p}_{E}^{k+j}, \text { for all } k\right\} .
$$

The parahoric subgroup $P \subset G L_{2 n}(F)$ is the units

$$
P=\mathcal{B}^{\times},
$$

and we let

$$
P_{0}=P ; \quad P_{j}=1+\mathcal{B}_{j}, \quad \text { for } \quad j \geq 1 .
$$

We define a function $\nu$ on $\mathfrak{g} l_{2 n}(F)$ by $\nu(X)=j$, where $j$ is the unique integer such that $X \in \mathcal{B}_{j} \backslash \mathcal{B}_{j+1}$. Note that if $X \in E$, then $\nu(X)=\operatorname{ord}_{E}(X)$. We embed $\mathfrak{g} l_{\left[E: E_{i}\right]}\left(E_{i}\right)$ in $\mathfrak{g} l_{n}\left(E_{0}\right)=\mathfrak{g} l_{n}(K) \subset \mathfrak{g} l_{2 n}(F)$ as the set of all elements of $\mathfrak{g} l_{n}(K)$ that centralize $E_{i} \subset E \subset \mathfrak{g} l_{n}(K)$. We will refer to this realization of $\mathfrak{g l} l_{\left[E: E_{i}\right]}\left(E_{i}\right)$ as $M_{i}$. In this situation, for $i=0, \ldots, r$, we will define

$$
\begin{aligned}
\mathcal{B}_{j}(i) & =\left\{X \in M_{i} \mid X \mathfrak{p}_{E_{i}}^{k} \subset \mathfrak{p}_{E_{i}}^{k+j}, \text { for all } k\right\}=\mathcal{B}_{j} \cap M_{i}, \\
P_{j}(i) & =P_{j} \cap M_{i},
\end{aligned}
$$

and

$$
\mathcal{B}(i)=\mathcal{B}_{0}(i), \quad P(i)=P_{0}(i)=\mathcal{B}(i) \cap P .
$$

The only difference from the definitions of $[\mathrm{MR}]$ is that here $\mathcal{B}_{j}(0)=\mathcal{B}_{j} \cap \mathfrak{g} l_{n}(K) \subsetneq$ $\mathcal{B}_{j}$ (since $\left.E_{0}=K\right)$, while in the previous paper $E_{0}=F$ and $\mathcal{B}_{j}(0)=\mathcal{B}_{j}$.

Lemma 3.1. ([MR], Corollary 3.5). For $0 \leq i \leq r$,

(i) $\varphi\left(M_{i}\right)=M_{i}$,

(ii) $\varphi\left(\mathcal{B}_{j}(i)\right)=\mathcal{B}_{j}(i), j \in \mathbb{Z}$,

(iii) $\varphi\left(P_{j}(i)\right)=P_{j}(i), j \geq 0$. 
For $1 \leq i \leq r$, write $\ell_{i}=\left[\frac{f_{E}\left(\theta_{i} \circ N_{E / E_{i}}\right)}{2}\right]$. Set

$$
\begin{aligned}
H_{0} & =E^{\times} P_{\ell_{r}}(r-1) \cdots P_{\ell_{2}}(1) P_{\ell_{1}}(0), \\
\mathcal{K}_{i} & =P_{\ell_{r}}(r-1) \cdots P_{\ell_{i+1}}(i), \quad 0 \leq i \leq r-1 ; \quad \mathcal{K}_{r}=\{1\}, \\
\mathcal{L}_{i} & =P_{\ell_{i}}(i-1) \cdots P_{\ell_{1}}(0), \quad 1 \leq i \leq r .
\end{aligned}
$$

If $H, K_{i}, L_{i}$ are the corresponding subgroups defined in $\S 3$ of [MR], then we note that $H_{0}=H \cap G, \mathcal{K}_{i}=K_{i} \cap G, \mathcal{L}_{i}=L_{i} \cap G$. For any subset $A \subset \mathfrak{g} l_{n}(K)$, we will write $A^{\varphi}$ for the $\varphi$-fixed points in $A$.

Lemma 3.2. (i) ([MR], Corollary 3.8). Let $x \in H_{0}^{\varphi}$, and $1 \leq i \leq r$. Then there exist $y \in\left(E^{\times} \mathcal{K}_{i}\right)^{\varphi}$ and $z \in \mathcal{L}_{i}$ such that $x=y z$.

(ii) ([MR], Lemma 3.9). Let $0 \leq i \leq r, j \geq 1$, and $\tau \in\left(H_{0} \cap M_{i}\right)^{\varphi}$. Then the map $x \mapsto x \tau \varphi(x)$ from $P_{j}(i)$ to $\left(\tau P_{j}(i)\right)^{\varphi}$ is onto.

\section{GOLDBERG'S REDUCIBILITY CRITERION}

Suppose that $\widetilde{\omega}$ is a character of $K^{\times}$of the form $\widetilde{\omega}(z)=\omega(z / \bar{z}), z \in K^{\times}$, for some character $\omega$ of the kernel $K^{1}$ of $N_{K / F}$. Let $C(G, \widetilde{\omega})$ be the space of locally constant complex-valued functions on $G$ which are compactly supported modulo the centre $Z_{K}$ of $G$, and satisfy $f(z g)=\widetilde{\omega}^{-1}(z) f(g), z \in Z_{K}, g \in G$. Let $Z_{F}$ denote the $F$-scalar matrices in $G$. Given $x \in G$, let

$$
G_{x \eta, Z_{F}}=\left\{g \in G \mid g x \eta\left(g^{-1}\right) x^{-1} \in Z_{F}\right\} .
$$

If $f \in C(G, \widetilde{\omega})$ and $x$ is $\eta$-semisimple, that is, $(x, \eta)$ is a semisimple element of $G \rtimes\langle\eta\rangle$, the $\eta$-twisted orbital integral of $f$ at $x$ is defined by ([G2], Def 1.9):

$$
\Phi_{\eta}(x, f)=\int_{G / G_{x \eta, Z_{F}}} f\left(g x \eta\left(g^{-1}\right)\right) d g^{\times},
$$

where $d g^{\times}$is the $G$-invariant measure on the quotient coming from Haar measures on $G$ and $G_{x \eta, Z_{F}}$.

Let $G^{\prime}$, resp. $G^{\prime \prime}$, be the $F$-rational points of the quasi-split unitary group in $2 n$, resp. $2 n+1$, variables defined with respect to $K / F$. Let $P^{\prime}$, resp. $P^{\prime \prime}$, be a maximal parabolic subgroup of $G^{\prime}$, resp. $G^{\prime \prime}$, having Levi component isomorphic to $G$, resp. $G \times K^{1}$ (see [G2], $\left.\S \S 2,6\right)$. Let $\pi$ be an irreducible supercuspidal representation of $G$. Given a character $\xi$ of $K^{1}$, define a supercuspidal representation $\Pi_{\xi}$ of $G \times K^{1}$ by

$$
\Pi_{\xi}(x, \alpha)=\pi(x) \xi\left(\operatorname{det}_{0}(x \eta(x)) \alpha\right), \quad x \in G, \alpha \in K^{1} .
$$

Here $\operatorname{det}_{0}$ denotes the determinant on $G$. Extend $\pi$, resp. $\Pi_{\xi}$, trivially across the unipotent radical to obtain a representation $\pi \otimes 1$, resp. $\Pi_{\xi} \otimes 1$, of $P^{\prime}$, resp. $P^{\prime \prime}$. Set $I(\pi)=\operatorname{Ind}_{P^{\prime}}^{G^{\prime}}(\pi \otimes 1)$ and $I\left(\Pi_{\xi}\right)=\operatorname{Ind}_{P^{\prime \prime}}^{G^{\prime \prime}}\left(\Pi_{\xi} \otimes 1\right)$. When $n=1, I(\pi)$ and $I\left(\Pi_{\xi}\right)$ are principal series representations, and it is known when such representations are reducible ([K1], [K2]). Thus we will assume that $n \geq 2$.

Let $h_{1}$ and $h_{2}$ be inequivalent hermitian matrices in $G$. Then $h_{1}$ is stably $\eta$ conjugate to $h_{2}$ ([G2], Definition 1.3, Corollary 1.7). This implies ([R]) that $G_{h_{1} \eta, Z_{F}}$ is (the $F$-rational points of) an inner form of $G_{h_{2} \eta, Z_{F}}$. Using an inner twisting, we define compatible measures on $G_{h_{1} \eta, Z_{F}}$ and $G_{h_{2}, \eta, Z_{F}}$, and hence on the quotients $G / G_{h_{k} \eta, Z_{F}}, k=1,2$. 
Theorem 4.1. ([G2], Theorem 2.9, Theorem 6.3) Let $\pi$ be an irreducible unitary supercuspidal representation of $G$ such that $\pi \circ \eta \sim \pi$. Then the following are equivalent:

(i) $I(\pi)$ is reducible.

(ii) $I\left(\Pi_{\xi}\right)$ is irreducible (for any $\xi$ ).

(iii) $\Phi_{\eta}\left(h_{1}, f\right)+\Phi_{\eta}\left(h_{2}, f\right)=0$ for every matrix coefficient $f$ of $\pi$.

Remark 4.2.

(1) The condition $\pi \circ \eta \sim \pi$ is necessary for reducibility of either of $I(\pi)$ and $I\left(\Pi_{\xi}\right)$ ([G2]).

(2) The sum $\Phi_{\eta}\left(h_{1}, f\right)+\Phi_{\eta}\left(h_{2}, f\right)$ can be expressed as a $\kappa$-twisted orbital integral of $f$ (see $\S 1$ of [G2]).

(3) The reducibility of $I\left(\Pi_{\xi}\right)$ is independent of the choice of $\xi([\mathrm{G} 2], \S 6)$.

As in $\S 2$, let $E$ be a tamely ramified degree $n$ extension of $K$, and take $\theta$ to be a unitary character of $E^{\times}$which is admissible over $K$ and satisfies $\theta^{-1}=\theta \circ \sigma$ for some $\sigma \in \operatorname{Aut}(E / F)$ such that $\sigma \mid K$ is non-trivial. Let $\pi$ be the irreducible supercuspidal representation of $G$ associated to $\theta$ via Howe's construction. Let $H_{0}$ be the open compact-mod-centre subgroup of $G$ defined in $\S 3$. Then $\pi=\operatorname{Ind}_{H_{0}}^{G} \kappa$ for some irreducible representation $\kappa$ of $H_{0}$. Let $\chi_{\kappa}$ denote the character of $\kappa$. Set

$$
\dot{\chi}_{\kappa}(x)= \begin{cases}\chi_{\kappa}(x), & \text { if } x \in H_{0}, \\ 0, & \text { otherwise. }\end{cases}
$$

Then the function $f_{\pi}$ defined by $f_{\pi}(x)=\dot{\chi}_{\kappa}\left(x h_{1}^{-1}\right)$ is a finite sum of matrix coefficients of $\pi$.

Let $h_{k}, k=1,2$, and $\varphi$ be as in $\S 3$. Recall that $h_{2}=a_{L} h_{1}$ and $a_{L} \in L^{\times}$, where $L=E^{\sigma}$. Then, noting that $\varphi(g)=h_{1} \eta\left(g^{-1}\right) h_{1}^{-1}$, we have

$$
\begin{aligned}
& \Phi_{\eta}\left(h_{1}, f_{\pi}\right)=\int_{G / G_{h_{1} \eta, Z_{F}}} \dot{\chi}_{\kappa}(g \varphi(g)) d g^{\times}, \\
& \Phi_{\eta}\left(h_{2}, f_{\pi}\right)=\int_{G / G_{h_{2} \eta, Z_{F}}} \dot{\chi}_{\kappa}\left(g a_{L} \varphi(g)\right) d g^{\times} .
\end{aligned}
$$

Our aim is to show that under certain conditions on $\theta$, both of the integrals $\Phi_{\eta}\left(h_{k}, f_{\pi}\right), k=1,2$, are positive and hence, by Theorem 4.1 , that $I(\pi)$ is irreducible, and $I\left(\Pi_{\xi}\right)$ is reducible.

\section{Preliminary Results}

Let the subgroups $H_{0}, \mathcal{K}_{i}, \mathcal{L}_{i}$, etc. be defined as in $\S 3$. For $0 \leq i \leq r$, let $\operatorname{det}_{i}: M_{i} \rightarrow E_{i}$ denote the determinant on $M_{i} \simeq \mathfrak{g} l_{\left[E: E_{i}\right]}\left(E_{i}\right)$. The notation tr will be used for the trace map on $\mathfrak{g l} l_{n}(K)$. Recall $([\mathrm{H}],[\mathrm{M}])$ that $\pi=\operatorname{Ind}_{H_{0}}^{G} \kappa$, where the inducing representation $\kappa$ is a tensor product:

$$
\kappa=\left(\Lambda \circ \operatorname{det}_{0}\right) \otimes \kappa_{1} \otimes \cdots \otimes \kappa_{r},
$$

and $\kappa_{i}$ is defined using the character $\theta_{i}$ of $E_{i}^{\times}$which appears in the Howe factorization of $\theta$. We continue to assume that $\Lambda$ and $\theta_{i}, 1 \leq i \leq r$, are chosen as in Lemma 2.3. When $f_{E}\left(\theta_{i} \circ N_{E / E_{i}}\right)>1$, the representation $\kappa_{i}$ is first defined on $E^{\times} \mathcal{K}_{i-1}$ and then extended across $\mathcal{L}_{i-1}$ by $\psi\left(\operatorname{tr}\left(c_{i}(\cdot-1)\right)\right)$ to get a representation of $H_{0}=E^{\times} \mathcal{K}_{i-1} \mathcal{L}_{i-1}$. Here, $c_{i} \in E_{i}$ is an element representing $\theta_{i}$ as in Lemma 2.3. 
If $f_{E}\left(\theta_{r}\right)=1$ then $\kappa_{r}$ is defined in terms of the cuspidal representation of the finite general linear group $P(r-1) / P_{1}(r-1)$ parametrized by $\theta_{r} \mid \mathcal{O}_{E}^{\times}$. This case will be discussed in $\S 7$.

Recall that $m_{i}=\left[\frac{f_{E}\left(\theta_{i} \circ N_{E / E_{i}}\right)+1}{2}\right]$ and $\ell_{i}=\left[\frac{f_{E}\left(\theta_{i} \circ N_{E / E_{i}}\right)}{2}\right], 1 \leq i \leq r$. If $i \leq r-1$ or if $i=r$ and $f_{E}\left(\theta_{r}\right)>1$, define a character $\omega_{i}$ of $E^{\times} \mathcal{K}_{i} P_{m_{i}}(i-1) \mathcal{L}_{i-1} \subset H_{0}$ by

$$
\omega_{i} \mid E^{\times} \mathcal{K}_{i}=\theta_{i} \circ \operatorname{det}_{i} \quad \text { and } \quad \omega_{i} \mid P_{m_{i}}(i-1) \mathcal{L}_{i-1}=\psi\left(\operatorname{tr}\left(c_{i}(\cdot-1)\right)\right) .
$$

The condition $2 m_{i} \geq f_{E}\left(\theta_{i} \circ N_{E / E_{i}}\right)$ guarantees that the two definitions coincide on the intersection $E^{\times} \mathcal{K}_{i} \cap P_{m_{i}}(i-1) \mathcal{L}_{i-1}([\mathrm{H}])$.

If $x \in H_{0}^{\varphi}$, then, by Lemma 3.2(i), $x \in L^{\times} P_{1}(0)$. For $x \in H_{0}^{\varphi}$, define

$$
\mu(x)= \begin{cases}1, & \text { if } x \in N_{E / L}\left(E^{\times}\right) P_{1}(0), \\ a_{L}, & \text { otherwise. }\end{cases}
$$

Lemma 5.1. ([MR], Lemma 5.1) If $E / L$ is ramified, then $f_{E}\left(\theta_{r}\right)>1$.

Lemma 5.2. ([MR], Lemma 5.2)

(i) Suppose that $x \in E^{\times} \mathcal{K}_{i} P_{m_{i}}(i-1) \mathcal{L}_{i-1}$ and $\varphi(x)=x$. If $f_{E}\left(\theta_{r}\right)=1$, make the additional assumption that $x \in E^{\times} P_{1}(0)$. Then $\omega_{i}(x)=\theta_{i}\left(N_{E / E_{i}}(\mu(x))\right)$.

(ii) If $x \in H_{0}^{\varphi}$, then $\Lambda\left(\operatorname{det}_{0}(x)\right)=\Lambda\left(N_{E / K}(\mu(x))\right)$.

The conductoral exponent $f_{E}\left(\theta_{i} \circ N_{E / E_{i}}\right)$ is even if and only if $m_{i}=\ell_{i}$. In this case, $E^{\times} \mathcal{K}_{i} P_{m_{i}}(i-1)=E^{\times} \mathcal{K}_{i-1}$, so $\omega_{i}$ is defined on all of $H_{0}$, and $\kappa_{i}=\omega_{i}$. In particular, if $m_{i}=\ell_{i}$, then $\operatorname{dim} \kappa_{i}=1$. If $i=r$ and $f_{E}\left(\theta_{r}\right)=1$, since the construction of $\kappa_{r}$ involves a cuspidal representation of a finite general group, we have $\operatorname{dim} \kappa_{r}>1$. Otherwise, $m_{i}=\ell_{i}+1 \geq 2$ and a Heisenberg construction is used to define $\kappa_{i}$ on $E^{\times} \mathcal{K}_{i}$, and $\operatorname{dim} \kappa_{i}>1$.

Proposition 5.3. If $\operatorname{dim} \kappa=1$ and $\theta \mid L^{\times} \equiv 1$, then $\Phi_{\eta}\left(h_{k}, f_{\pi}\right)>0, k=1,2$.

Proof. By the above remarks, $m_{i}=\ell_{i}, 1 \leq i \leq r$, and $\kappa_{i}=\omega_{i}$. If $x=g \varphi(g) \in H_{0}$, then $\varphi(x)=x$, so Lemma 5.2 applies and

$$
\begin{aligned}
& \kappa(g \varphi(g))=\Lambda\left(\operatorname{det}_{0}(g \varphi(g))\right) \prod_{i=1}^{r} \kappa_{i}(g \varphi(g)) \\
& =\Lambda\left(N_{E / K}(\mu(g \varphi(g)))\right) \prod_{i=1}^{r} \theta_{i}\left(N_{E / E_{i}}(\mu(g \varphi(g)))\right)=\theta(\mu(g \varphi(g))), \quad \text { if } g \varphi(g) \in H_{0} .
\end{aligned}
$$

Similarly, if $g a_{L} \varphi(g) \in H_{0}$, by Lemma 5.2,

$$
\kappa\left(g a_{L} \varphi(g)\right)=\Lambda\left(N_{E / K}\left(\mu\left(g a_{L} \varphi(g)\right)\right)\right) \prod_{i=1}^{r} \kappa_{i}\left(g a_{L} \varphi(g)\right)=\theta\left(\mu\left(g a_{L} \varphi(g)\right)\right) .
$$

Since $\mu(x) \in L^{\times}$for $x \in H_{0}^{\varphi}$ and $\theta \mid L^{\times} \equiv 1$, it follows from (4.2) that $\Phi_{\eta}\left(h_{k}, f_{\pi}\right)=$ $\Phi_{\eta}\left(h_{k}, \mathbf{1}_{H_{0} h_{1}}\right), k=1,2$, where $\mathbf{1}_{H_{0} h_{1}}$ denotes the characteristic function of $H_{0} h_{1}$. Since $P_{j}(0)$ and $a_{L} P_{j}(0)$ are contained in $H_{0}$ for sufficiently large $j$, it is a simple matter to show, using Lemma 3.2 (ii), that $\Phi_{\eta}\left(h_{k}, \mathbf{1}_{H_{0} h_{1}}\right)>0, k=1,2$.

We collect some results of $[\mathrm{MR}]$ which will be used later in this paper.

Lemma 5.4. ([MR], Lemmas 5.4-5.7)

(i) Suppose that $K \subset N_{1} \subset N_{2} \subset E, \sigma\left(N_{j}\right)=N_{j}, j=1,2$, and $N_{2} /\left(N_{2} \cap L\right)$ is ramified. Then $N_{1} /\left(N_{1} \cap L\right)$ is ramified and $e\left(N_{2} / N_{1}\right)$ is odd.

(ii) If $E / L$ is ramified, then $\operatorname{dim} \kappa=1$. 
(iii) If a Heisenberg construction is required for one of the $\kappa_{i}$ 's, then $E / L$ is unramified.

(iv) If $r>1, f_{E}\left(\theta_{r}\right)=1$, and $e\left(E_{r-1} /\left(E_{r-1} \cap L\right)\right)=2$, then $\operatorname{dim} \kappa_{i}=1$ for $1 \leq i \leq r-1$.

\section{The Heisenberg construction}

Fix $i, 1 \leq i \leq r$. Suppose that $f_{E}\left(\theta_{i} \circ N_{E / E_{i}}\right)$ is odd, that is, $m_{i}=\ell_{i}+1$. If $i=r$, assume in addition that $\ell_{r} \geq 1$. Recall that in this case (Lemma 5.4(iii)) $E / L$ must be unramified. Set

$$
\begin{aligned}
& H_{i}=K^{\times}\left(1+\mathfrak{p}_{E}\right)\left(\mathcal{K}_{i} P_{\ell_{i}}(i-1) \cap P_{1}(0)\right), \\
& H_{i}^{\prime}=K^{\times}\left(1+\mathfrak{p}_{E}\right)\left(\mathcal{K}_{i} P_{m_{i}}(i-1) \cap P_{1}(0)\right) .
\end{aligned}
$$

Let $\omega_{i}$ be the character of $E^{\times} \mathcal{K}_{i} P_{m_{i}}(i-1) \mathcal{L}_{i-1}$ defined in $\S 5$. Let $\chi_{i}$ denote the character of $\kappa_{i}$. A Heisenberg construction is used to define $\kappa_{i} \mid E^{\times} \mathcal{K}_{i} P_{\ell_{i}}(i-1)$ in such a way that the restriction of $\chi_{i}$ to $H_{i}^{\prime}$ is a multiple of $\omega_{i} \mid H_{i}^{\prime}$. Then, if $i \geq 2, \kappa_{i}$ is extended by $\psi\left(\operatorname{tr}\left(c_{i}(\cdot-1)\right)\right)$ on $\mathcal{L}_{i-1}$ to produce a representation of $H_{0}$. In this section, we see that, for $x \in\left(E^{\times} H_{i}\right)^{\varphi}, \chi_{i}(x)$ is a real scalar multiple of $\theta_{i}\left(N_{E / E_{i}}(\mu(x))\right)$. When the scalar multiple is non-zero, we compute its sign (Corollary 6.5).

If $F \subset N \subset E$, let $\zeta_{N}$ denote the set of roots of unity in $N$ of order prime to $p$. We assume that a uniformizer $\varpi_{N} \in N$ is chosen so that $\varpi_{N}^{e(N / F)} \in \varpi \zeta_{F}$, where $\varpi$ is a uniformizer in $F$. Let $C_{N}$ be the subgroup of $N^{\times}$generated by $\varpi_{N}$ and $\zeta_{N}$.

Lemma 6.1. Let $x \in L^{\times}\left(H_{i} \cap P_{1}(0)\right)$.

(i) There exists a unique $c_{L}(x) \in C_{L}$ such that $x \in c_{L}(x)\left(H_{i} \cap P_{1}(0)\right)$.

(ii) Suppose that $y^{-1} x y \in E^{\times} H_{i}^{\prime}$ for some $y \in E^{\times} H_{i}$. Then, given any subfield $N$ of $E$ containing $E_{0}=K$,

$$
y^{-1} x y \in N^{\times} H_{i}^{\prime} \Longleftrightarrow c_{L}(x) \in N^{\times} .
$$

Remark 6.2. In [MR], an analogue of the above lemma was proved for points which were $\varphi$-invariant, but the proof only required $x \in L^{\times}\left(H_{i} \cap P_{1}(0)\right)$.

Define

$$
\mathcal{S}_{i}=\left\{N \mid E_{i-1} \subset N \subset E, N \not \supset E_{i}\right\},
$$

To each $N \in \mathcal{S}_{i}$, there are attached a sign $\operatorname{sgn}(N) \in\{ \pm 1\}$, and a positive integer $D(N)$ as defined in (3.6.47) of [M]. Set

$$
\operatorname{sgn}(x)=\prod_{\left\{N \in \mathcal{S}_{i} \mid c_{L}(x) \notin N^{\times}\right\}} \operatorname{sgn}(N), \quad x \in L^{\times}\left(H_{i} \cap P_{1}(0)\right) .
$$

Let $q_{E_{i-1}}$ denote the cardinality of the residue class field of $E_{i-1}$.

Lemma 6.3. Let $x \in\left(E^{\times} H_{i}\right)^{\varphi}$. If $x$ is conjugate to an element of $E^{\times} H_{i}^{\prime}$, then

$$
\chi_{i}(x)=q_{E_{i-1}}^{\sum_{\left\{N \in \mathcal{S}_{i} \mid c_{L}(x) \in N^{\times}\right\}} D(N)} \operatorname{sgn}(x) \theta_{i}\left(N_{E / E_{i}}(\mu(x))\right) .
$$

Otherwise $\chi_{i}(x)=0$. Here, $\mu$ is as defined in $\S 5$.

Proof. The second statement of the lemma follows from $[\mathrm{M}], \S 3.6$. Thus, without loss of generality, we assume that there exists $y \in E^{\times} H_{i}$ such that $y^{-1} x y \in E^{\times} H_{i}^{\prime}$. 
Let $\omega_{i}$ and $\mu$ be defined as in $\S 5$. It follows from results of [M] (see Lemma 6.1 of $[\mathrm{MR}])$ and Lemma 6.1, that

$$
\chi_{i}(x)=q_{E_{i-1}}^{\sum_{\left\{N \in \mathcal{S}_{i} \mid c_{L}(x) \in N^{\times}\right\}} D(N)} \operatorname{sgn}(x) \omega_{i}\left(y^{-1} x y\right) .
$$

To complete the proof, arguing as for Lemma 6.4 of [MR] results in:

$$
\omega_{i}\left(y^{-1} x y\right)=\theta_{i}\left(N_{E / E_{i}}(\mu(x))\right) \text {. }
$$

Lemma 6.4. Let $L^{\prime}=L_{u n}\left(\varpi_{L} \sqrt{\varepsilon}\right)$, where $L_{u n}$ is the maximal unramified extension of $F$ contained in $L$ and $\varepsilon$ is a non-square in $\zeta_{L_{u n}}$. Suppose that $N \in \mathcal{S}_{i}$ and $\sigma(N)=N$.

(i) If $K / F$ is unramified, then $\operatorname{sgn}(N)=1$.

(ii) If $K / F$ is ramified and $e(E / K)$ is even, then $\operatorname{sgn}(N)=1$.

(iii) If $K / F$ is ramified, $e(E / K)$ is odd, and $e\left(E_{i-1} /\left(E_{i-1} \cap L\right)\right)=e\left(E_{i} /\left(E_{i} \cap L\right)\right)$, then $\operatorname{sgn}(N)=1$.

(iv) If $K / F$ is ramified, $e(E / K)$ is odd, $e\left(E_{i} /\left(E_{i} \cap L\right)\right)=1$ and $e\left(E_{i-1} /\left(E_{i-1} \cap L\right)\right)$ $=2$, then

$$
\operatorname{sgn}(N)= \begin{cases}-1, & \text { if } N=L^{\prime} \\ 1, & \text { otherwise }\end{cases}
$$

Proof. As shown in Proposition 3.6.55 of $[\mathrm{M}], \operatorname{sgn}(N)=1$ whenever $f(E / N)>2$. By arguing as in the second part of the proof of Lemma 7.4 of $[\mathrm{MR}]$, we see that $\operatorname{sgn}(N)=1$ whenever $f(E / N)=1$. Thus we need only consider the case $f(E / N)=$ 2 .

Suppose that $K / F$ is unramified. As $E / L$ is also unramified, and $K \not \subset L$, we have $f(E / K)=f(L / F)$ odd. In particular, as $K \subset N, f(E / N)$ must be odd, and so (i) follows.

Suppose that $K / F$ is ramified. Assume that $N \in \mathcal{S}_{i}, f(E / N)=2$ and $\sigma(N)=$ $N$. Then, by Proposition 7.6 of [MR], $\operatorname{sgn}(N)=1$ if $[E: N]>2$, and $\operatorname{sgn}(N)=-1$ if $[E: N]=2$. By Lemma 7.5(i) of [MR], $L$ and $L^{\prime}$ are the only two extensions $N^{\prime}$ of $F$ in $E$ satisfying $\sigma\left(N^{\prime}\right)=N^{\prime}$ and $\left[E: N^{\prime}\right]=f\left(E / N^{\prime}\right)=2$. By Lemma 7.5(ii) of [MR], $K \subset E_{i-1} \subset L^{\prime}$ is equivalent to $e(E / K)$ odd and $e\left(E_{i-1} /\left(E_{i-1} \cap L\right)\right)=2$. Also, if $e(E / K)$ is odd, then $L^{\prime} \not \supset E_{i}$ is equivalent to $e\left(E_{i} /\left(E_{i} \cap L\right)\right)=1$. Thus, by definition of $\mathcal{S}_{i}, L^{\prime} \in \mathcal{S}_{i}$ is equivalent to the three conditions $e(E / K)$ odd, $e\left(E_{i-1} /\left(E_{i-1} \cap L\right)\right)=2$ and $e\left(E_{i} /\left(E_{i} \cap L\right)\right)=1$. Parts (ii)-(iv) now follow.

Corollary 6.5. Let $x \in L^{\times}\left(H_{i} \cap P_{1}(0)\right)$. Then, if $\nu$ is as defined in $\S 3$,

$$
\operatorname{sgn}(x)=\left\{\begin{array}{cc}
(-1)^{\nu(x)}, & \text { if } e(E / K) \text { is odd, } e\left(E_{i-1} /\left(E_{i-1} \cap L\right)\right)=2, \\
\text { and } e\left(E_{i} /\left(E_{i} \cap L\right)\right)=1, & \text { otherwise. }
\end{array}\right.
$$

Proof. First note that if $\sigma(N) \neq N$, then $\sigma\left(E_{i-1}\right)=E_{i-1}$ implies that $E_{i-1} \subset$ $\sigma(N)$. Also, $E_{i} \not \subset N$ and $\sigma\left(E_{i}\right)=E_{i}$ implies that $E_{i} \not \subset \sigma(N)$. Thus if $\sigma(N) \neq N$, we have $N \in \mathcal{S}_{i}$ if and only if $\sigma(N) \in \mathcal{S}_{i}$. It follows easily from the definitions in $[\mathrm{M}]$ that $\operatorname{sgn}(N)=\operatorname{sgn}(\sigma(N))$. Therefore, when computing $\operatorname{sgn}(x)$, we need only consider those $N \in \mathcal{S}_{i}$ such that $c_{L}(x) \notin N^{\times}$and $\sigma(N)=N$.

It now follows from Lemma 6.4 that we need only consider the case where $e(E / K)$ is odd, $e\left(E_{i-1} /\left(E_{i-1} \cap L\right)\right)=2$, and $e\left(E_{i} /\left(E_{i} \cap L\right)\right)=1$. (Note that in this case 
$K / F$ is ramified, by Lemma 5.4(i)). By Lemma 6.4(iv),

$$
\operatorname{sgn}(x)= \begin{cases}-1, & \text { if } c_{L}(x) \notin L^{\prime \times} \\ 1, & \text { if } c_{L}(x) \in L^{\prime \times}\end{cases}
$$

By definition, $L^{\prime}$ is a quadratic extension of $L_{u n}\left(\varpi_{L}^{2}\right)$ containing $\zeta_{L}$ and not containing $\varpi_{L}$. It is immediate that $c_{L}(x) \in L^{\prime \times}$ if and only if $c_{L}(x) \in \varpi_{L}^{2 k} \zeta_{L}$ for some integer $k$; that is, if and only if $\nu\left(c_{L}(x)\right)=\nu(x)$ is even.

We can predict precisely when there will be a Heisenberg construction with $\operatorname{sgn}(x)=-1$ for some $x$, as follows:

Lemma 6.6. Assume that $E / L$ is unramified.

(i) Suppose that $K / F$ is ramified and $e(E / K)$ is odd. Then there exists a unique $j, 1 \leq j \leq r$, having the property that $m_{j}=\ell_{j}+1$ and $\operatorname{sgn}(x)=(-1)^{\nu(x)}$, $x \in L^{\times}\left(H_{j} \cap P_{1}(0)\right)$. In particular, for all $i \neq j, 1 \leq i \leq r$, such that $m_{i}=\ell_{i}+1$, we have $\operatorname{sgn}(x)=1$, for every $x \in L^{\times}\left(H_{i} \cap P_{1}(0)\right)$.

(ii) If the conditions of (i) are not satisfied, then for all $i, 1 \leq i \leq r$, such that $m_{i}=\ell_{i}+1$, we have $\operatorname{sgn}(x)=1$, for every $x \in L^{\times}\left(H_{i} \cap P_{1}(0)\right)$.

Proof. First suppose that $K / F$ is ramified and $e(E / K)$ is odd. Then, by Lemma $5.4(\mathrm{i})$, there exists a unique $j, 1 \leq j \leq r$, such that $e\left(E_{j} /\left(E_{j} \cap L\right)\right)=1$ and $e\left(E_{j-1} /\left(E_{j-1} \cap L\right)\right)=2$. As $e\left(E / E_{j}\right)$ is odd, it follows from

$$
f_{E}\left(\theta_{j} \circ N_{E / E_{j}}\right)=e\left(E / E_{j}\right)\left(f_{E_{j}}\left(\theta_{j}\right)-1\right)+1
$$

that $m_{j}=\ell_{j}+1$ if and only if $f_{E_{j}}\left(\theta_{j}\right)$ is odd. To show that $f_{E_{j}}\left(\theta_{j}\right)$ is odd, argue as in the proof of Corollary 7.11 of $[\mathrm{MR}]$. All statements concerning $\operatorname{sgn}(x)$ are now immediate consequences of Corollary 6.5.

\section{The CASe $f_{E}\left(\theta_{r}\right)=1$.}

Throughout this section, we assume that $f_{E}\left(\theta_{r}\right)=1$ and that if $r>1$, then $\kappa_{j}$ is one-dimensional for $1 \leq j \leq r-1$. Using a modification of the arguments of $\S 10$ of [MR], we express each $\Phi_{\eta}\left(h_{k}, f_{\pi}\right), k=1,2$, in terms of sums of the character $\chi_{r}$ of $\kappa_{r}$ over subsets of $\bar{H}_{0}$. Certain conditions on $\theta$ imply that $\Phi_{\eta}\left(h_{k}, f_{\pi}\right)>0$. Omitting some of the details, we indicate how to adapt the results of $\S 10$ of [MR] to this setting.

We now define prime elements in $E, L, E_{r-1}$ and $E_{r-1} \cap L$ as in [MR]. Recall that $f_{E}\left(\theta_{r}\right)=1$ implies that $E$ is unramified over $L$ (Lemma 5.1) and over $E_{r-1}$. Set $e_{0}=e\left(E_{r-1} / E_{r-1} \cap L\right)$ and $f_{0}=f\left(E_{r-1} / E_{r-1} \cap L\right)$. Fix a prime element $\varpi_{0}$ in $E_{r-1} \cap L$ and a non-square root of unity $\varepsilon$ in $L$. If $e_{0}=1$, then $E /\left(E_{r-1} \cap L\right)$ is unramified and we choose prime elements in $E$ and $L$ as follows: $\varpi_{E}=\varpi_{L}=\varpi_{0}$. If $e_{0}=2$, then $\varpi_{E}=\sqrt{\varpi_{0}}$ is a prime element in $E$ which generates $E_{r-1}$ over $E_{r-1} \cap L$ and satisfies $\sigma\left(\varpi_{E}\right)=-\varpi_{E}$. Furthermore, the element $\varpi_{L}=\sqrt{\varepsilon \varpi_{0}}=\sqrt{\varepsilon} \varpi_{E}$ is a prime element in $L$.

Let $\bar{M}$ denote the residue class field of a $p$-adic field $M$. Set

$$
\bar{H}_{0}=\left(H_{0} \cap P(0)\right) /\left(H_{0} \cap P_{1}(0)\right) .
$$

It follows from the definition of $H_{0}$ that

$$
\bar{H}_{0} \simeq P(r-1) / P_{1}(r-1) \simeq G L_{\left[E: E_{r-1}\right]}\left(\bar{E}_{r-1}\right) .
$$

If $r>1$, then $\bar{H}_{0}=\bar{H}$, where $\bar{H}=(H \cap P) /\left(H \cap P_{1}\right)$ is as in $\S 9$ of [MR]. If $r=1$, then since $E_{0}=K$ here and the $E_{0}$ of $[\mathrm{MR}]$ was $F$, we have $\bar{H}_{0}=\bar{H} \cap G L_{n}(\bar{K})$. 
We can now apply the results of $\S 9$ of [MR], remembering to replace $\bar{H}$ by $\bar{H}_{0}$ in the case $r=1$.

As $f_{E}\left(\theta_{r}\right)=1$ and $\theta_{r}$ is generic over $E_{r-1}$, the character $\theta_{r} \mid \mathcal{O}_{E}^{\times}$determines a character of $\bar{E}^{\times}$which corresponds to an irreducible cuspidal representation $\bar{\kappa}_{r}$ of $\bar{H}_{0}$. The restriction of $\kappa_{r}$ to $H_{0} \cap P(0)$ is trivial on $H_{0} \cap P_{1}(0)$ and induces $\bar{\kappa}_{r}$ on $\bar{H}_{0}$. As the prime element $\varpi_{E}$ above is a prime element in $E_{r-1}$, setting $\kappa_{r}\left(\varpi_{E}\right)=\theta_{r}\left(\varpi_{E}\right) \kappa_{r}(1)$ extends $\kappa_{r}$ to $H_{0}$.

Let $\mathcal{C}_{\bar{E}}$, resp. $\mathcal{C}_{\bar{L}}$, be the set of elements in $\bar{H}_{0}$ whose semisimple part is conjugate to an element of $\bar{E}$, resp. $\bar{L}$. Next, define $\mathcal{S}_{E-L}$, resp. $\mathcal{S}_{L}$, to be the set of $x \in$ $H_{0} \cap P(0)$ such that the image of $x$ in $\bar{H}_{0}$ belongs to $\mathcal{C}_{\bar{E}} \backslash \mathcal{C}_{\bar{L}}$, resp. $\mathcal{C}_{\bar{L}}$. It follows from properties of the cuspidal representation $\bar{\kappa}_{r}$ of $\bar{H}_{0}$ that if $x \in H_{0} \cap P(0)$ does not belong to $\mathcal{S}_{L} \cup \mathcal{S}_{E-L}$, then $\chi_{r}(x)=0$. As we will see in Lemma 7.2 , we need only consider values of $\chi_{r}$ for $x \in\left(\varpi_{E}^{k}\left(H_{0} \cap P(0)\right)\right)^{\varphi}, k=1,2$. The following lemma gives information on properties of such $x$, when $\varpi_{E}^{-k} x \in \mathcal{S}_{L} \cup \mathcal{S}_{E-L}, k=1,2$.

\section{Lemma 7.1.}

(i) Suppose that $x \in P(r-1)^{\varphi}$. Then there exists $g \in P(r-1)$ such that $x=g \varphi(g)$.

(ii) Suppose that $x \in\left(\varpi_{E} P(r-1)\right)^{\varphi}$. If $e_{0}=2$ and $\varpi_{E}^{-1} x \in \mathcal{S}_{E-L}$, or if $e_{0}=1$, then there exists $g \in P(r-1)$ such that $x=g \varpi_{L} \varphi(g)$.

(iii) Suppose that $e_{0}=2$ and $f\left(L /\left(E_{r-1} \cap L\right)\right)$ is even. Fix $\delta \in P(r-1) \cap \mathcal{S}_{L}$ such that $\varphi\left(\varpi_{E} \delta\right)=\varpi_{E} \delta$. If $x \in\left(\varpi_{E} P(r-1)\right)^{\varphi}$ and $\varpi_{E}^{-1} x \in \mathcal{S}_{L}$, then there exists $g \in P(r-1)$ such that $x=g \varpi_{E} \delta \varphi(g)$. Furthermore, $x=g_{1} \varphi\left(g_{1}\right)$ for some $g_{1} \in G$.

Proof. Statements (i), (ii), and the first part of (iii) are proved as in Lemma 10.3 of $[\mathrm{MR}]$.

Recall that $h_{2}=\varpi_{L} h_{1}$ (§3). Given $y \in G, y \in G^{\varphi}$ if and only if $y h_{1}$ is hermitian. Recall ( $(3)$ that $h_{1}$ and $h_{2}$ belong to distinct equivalence classes of hermitian matrices. It follows that $G^{\varphi}$ is the disjoint union of the sets $\left\{g \varpi_{L}^{\ell} \varphi(g) \mid g \in G\right\}, \ell=0,1$, the elements of the first set, resp. second set, having determinants in $N_{K / F}\left(K^{\times}\right)$, resp. in $N_{E / K}\left(\varpi_{L}\right) N_{K / F}\left(K^{\times}\right)=F^{\times} \backslash N_{K / F}\left(K^{\times}\right)$. Assume that $\delta$ is as in (iii). As $\varpi_{E} \delta \in G^{\varphi}$ by assumption, to show that $\varpi_{E} \delta=y \varphi(y)$ for some $y \in G$, it suffices to show that $\operatorname{det}_{0}\left(\varpi_{E} \delta\right) \in N_{K / F}\left(K^{\times}\right)$.

Because $\delta \in \mathcal{S}_{L}$,

$$
\operatorname{det}_{0}(\delta) \in \operatorname{det}_{0}\left(\mathcal{O}_{L}^{\times}\right)=\operatorname{det}_{0}\left(N_{E / L}\left(\mathcal{O}_{E}^{\times}\right)\right) \subset N_{K / F}\left(\mathcal{O}_{K}^{\times}\right) .
$$

Also, by choice of the prime element $\varpi_{E} \in E_{r-1}$, since

$$
\left[E: E_{r-1}\right]=2 f\left(L /\left(E_{r-1} \cap L\right)\right)
$$

is divisible by $4, N_{E / E_{r-1}}\left(\varpi_{E}\right)=\operatorname{det}_{r-1}\left(\varpi_{E}\right)=\varpi_{E}^{\left[E: E_{r-1}\right]} \in\left(E_{r-1} \cap L^{\times}\right)^{2}$. Thus $\operatorname{det}_{0}\left(\varpi_{E}\right)=N_{E / K}\left(\varpi_{E}\right) \in\left(F^{\times}\right)^{2}$. We conclude that $\operatorname{det}_{0}\left(\varpi_{E} \delta\right) \in N_{K / F}\left(K^{\times}\right)$. Thus $\varpi_{E} \delta=y \varphi(y)$, for some $y \in G$. Taking $x$ as in (iii), there exists $g \in P(r-1)$ such that $x=g \varpi_{E} \delta \varphi(g)=g y \varphi(g y)$. Set $g_{1}=g y$.

Let $\mathcal{F}_{k}=f_{\pi} \cdot \mathbf{1}_{\left(H_{0} \cap P(0)\right) h_{k}}, k=1,2$, where we write $\mathbf{1}_{S}$ for the characteristic function of a subset $S$ of $G$.

Lemma 7.2. Set $e=e(E / F)$. Let $(\cdot, \cdot)$ denote gcd.

(i) $\Phi_{\eta}\left(h_{1}, f_{\pi}\right)=\frac{e}{(2, e)}\left(\Phi_{\eta}\left(h_{1}, \mathcal{F}_{1}\right)+\Phi_{\eta}\left(h_{1}, \mathcal{F}_{2}\right)\right)$.

(ii) $\Phi_{\eta}\left(h_{2}, f_{\pi}\right)=\frac{e}{(2, e)} \Phi_{\eta}\left(h_{2}, \mathcal{F}_{2}\right)$. 
Proof. By arguing as in the proof of Lemma 10.1 of $[\mathrm{MR}]$,

$$
\chi_{\kappa}\left(\varpi_{E}^{j} x \varphi\left(\varpi_{E}^{j}\right)\right)=\chi_{\kappa}(x), \quad x \in H_{0} .
$$

Let $C_{k}=\left\{g \varpi_{L}^{k-1} \varphi(g) \mid g \in G\right\}, k=1,2$. Recall (see above) that $G^{\varphi}$ is the disjoint union of $C_{1}$ and $C_{2}$. Given $j \in \mathbb{Z}$ and $\alpha \in \mathcal{O}_{E}^{\times}$, define a map $\lambda_{\alpha, j}$ from $G$ to $G$ by $\lambda_{\alpha, j}(x)=\varpi_{E}^{j} \alpha x \varphi\left(\varpi_{E}^{j} \alpha\right)$. For $1 \leq k, \ell \leq 2$, the map $\lambda_{\alpha, j}$ restricts to a measure-preserving bijection between

$$
C_{k} \cap \varpi_{E}^{\ell-1}\left(H_{0} \cap P(0)\right) \quad \text { and } \quad C_{k} \cap \varpi_{E}^{\ell-1+2 j}\left(H_{0} \cap P(0)\right),
$$

where the measure is the one on $G / G_{h_{k} \eta, Z_{F}}$. Thus, using the map $\lambda_{\alpha, j}$, and the fact that $\chi_{\kappa} \circ \lambda_{\alpha, j}=\chi_{\kappa} \circ \lambda_{\alpha, 0}$ (see above),

$$
\begin{aligned}
& \int_{G / G_{h_{k} \eta, Z_{F}}}\left(\dot{\chi}_{\kappa} \mathbf{1}_{\varpi_{E}^{\ell-1}\left(H_{0} \cap P(0)\right)}\right)\left(g \varpi_{L}^{k-1} \varphi(g)\right) d g^{\times} \\
& =\int_{G / G_{h_{k} \eta, Z_{F}}} \dot{\chi}_{\kappa}\left(\alpha g \varpi_{L}^{k-1} \varphi(\alpha g)\right) \mathbf{1}_{\varpi_{E}^{\ell-1+2 j}\left(H_{0} \cap P(0)\right)}\left(g \varpi_{L}^{k-1} \varphi(g)\right) d g^{\times} \\
& =\int_{G / G_{h_{k} \eta, Z_{F}}}\left(\dot{\chi}_{\kappa} \mathbf{1}_{\varpi_{E}^{\ell-1+2 j}\left(H_{0} \cap P(0)\right)}\right)\left(g \varpi_{L}^{k-1} \varphi(g)\right) d g^{\times} .
\end{aligned}
$$

To obtain the second equality, we have used the fact that $\lambda_{\alpha, 0}$ fixes the set $\varpi_{E}^{\ell-1+2 j}\left(H_{0} \cap P(0)\right)$.

The smallest positive integer $j$ such that $N_{E / L}\left(\varpi_{E}^{j} \mathcal{O}_{E}^{\times}\right) \cap F^{\times} \neq \emptyset$, that is, such that $\varpi_{E}^{j} \mathcal{O}_{E}^{\times} \cap G_{h_{k} \eta, Z_{F}} \neq \emptyset$, is $j=e /(2, e)$. Therefore, applying (7.1) (which is independent of the choice of $\alpha \in \mathcal{O}_{E}^{\times}$), we conclude from (4.1) and $H_{0}=$ $\bigcup_{j \in \mathbb{Z}} \varpi_{E}^{j}\left(H_{0} \cap P(0)\right)$, that

$$
\begin{array}{r}
\Phi_{\eta}\left(h_{k}, f_{\pi}\right)=\frac{e}{(2, e)} \sum_{1 \leq \ell \leq 2} \int_{G / G_{h_{k} \eta, Z_{F}}}\left(\dot{\chi}_{\kappa} \mathbf{1}_{\varpi_{E}^{\ell-1}\left(H_{0} \cap P(0)\right)}\right)\left(g \varpi_{L}^{k-1} \varphi(g)\right) d g^{\times}, \\
k=1,2 .
\end{array}
$$

As $h_{2}=\varpi_{L} h_{1}, \varpi_{L}$ normalizes $H_{0} \cap P(0)$, and $\varpi_{E} \in \varpi_{L} \mathcal{O}_{E}^{\times} \subset \varpi_{L}\left(H_{0} \cap P(0)\right)$, it follows that $\left(H_{0} \cap P(0)\right) h_{2}=\varpi_{E}\left(H_{0} \cap P(0)\right) h_{1}$. Therefore (see comments preceding

$$
\Phi_{\eta}\left(h_{k}, \mathcal{F}_{\ell}\right)=\int_{G / G_{h_{k} \eta, Z_{F}}}\left(\dot{\chi}_{\kappa} \mathbf{1}_{\varpi_{E}^{\ell-1}\left(H_{0} \cap P(0)\right)}\right)\left(g \varpi_{L}^{k-1} \varphi(g)\right) d g^{\times}, \quad k, \ell=1,2 .
$$

Comparing this with the above expression for $\Phi_{\eta}\left(h_{k}, f_{\pi}\right)$, we see that it remains to show that $\Phi_{\eta}\left(h_{2}, \mathcal{F}_{1}\right)=0$.

Let $x \in\left(H_{0} \cap P(0)\right)^{\varphi}$. By Lemma 3.2(i), there exists $y \in\left(E^{\times} \mathcal{K}_{r-1}\right)^{\varphi}=$ $\left(E^{\times} P(r-1)\right)^{\varphi}$ and $z \in \mathcal{L}_{r-1}$ such that $x=y z$. As $x \in P(0)$ and $z \in P_{1}(0)$, it follows that $y \in P(r-1)^{\varphi}$. By Lemma 7.1(i), there exists $y_{1} \in P(r-1)$ such that $y=y_{1} \varphi\left(y_{1}\right)$. Since $z \in P_{1}(0)$, and $x=y z$ and $y$ are $\varphi$-invariant, it follows that $\operatorname{det}_{0}(z) \in 1+\mathfrak{p}_{F}$. Thus $\operatorname{det}_{0}(x) \in N_{K / F}\left(\operatorname{det}_{0}\left(y_{1}\right)\right)\left(1+\mathfrak{p}_{F}\right) \subset N_{K / F}\left(K^{\times}\right)$. Since $x \in C_{1} \cup C_{2}$, $\operatorname{det}_{0}\left(C_{1}\right) \subset N_{K / F}\left(K^{\times}\right)$, and $\operatorname{det}_{0}\left(C_{2}\right) \subset F^{\times} \backslash N_{K / F}\left(K^{\times}\right)$, we must have $x \in C_{1}$. It follows from $\left(H_{0} \cap P(0)\right)^{\varphi} \subset C_{1}, C_{1} \cap C_{2}=\emptyset$, and (7.2) that $\Phi_{\eta}\left(h_{2}, \mathcal{F}_{1}\right)=0$. 
As the $\kappa_{i}$ 's, $1 \leq i \leq r-1$, are one-dimensional, their values on the relevant $\varphi$-invariant elements in $\varpi_{E}^{j}\left(H_{0} \cap P(0)\right), j=1,2$, are easily computed in terms of the characters $\theta_{i}$. In [MR], this was done in Lemma 10.2. Here, the result still holds, and it is proved the same way (with $\bar{H}_{0}$ replacing $\bar{H}$ ). The computation for $\Lambda$ is handled in exactly the same way. Combining this with the definition of $\kappa_{r}$ we get, for $x \in\left(H_{0} \cap P(0)\right)^{\varphi} \cup\left(\varpi_{E}\left(H_{0} \cap P(0)\right)\right)^{\varphi}$,

$$
\chi_{\kappa}(x)= \begin{cases}\theta\left(\varpi_{E}^{\nu(x)}\right) \chi_{r}\left(\varpi_{E}^{-\nu(x)} x\right), & \text { if } \varpi_{E}^{-\nu(x)} x \in \mathcal{S}_{L}, \\ \theta\left(\varpi_{L}\right) \theta_{r}(\sqrt{\varepsilon})^{-1} \chi_{r}\left(\varpi_{E}^{-1} x\right), & \text { if } \nu(x)=1 \text { and } \varpi_{E}^{-1} x \in \mathcal{S}_{E-L}, \\ 0, & \text { if } \varpi_{E}^{\nu(x)} x \notin \mathcal{S}_{L} \cup \mathcal{S}_{E-L} .\end{cases}
$$

Let $c=(-1)^{f_{0}}$. For $x \in\left(H_{0} \cap P(0)\right)^{\varphi} \cup\left(\varpi_{E}\left(H_{0} \cap P(0)\right)\right)^{\varphi}$, observe that the image of $\varpi_{E}^{-\nu(x)} x$ in $\bar{H}_{0}$ belongs to $\bar{H}_{0}^{\varphi}$ if $\nu(x)=0$, and to $\bar{H}_{0}^{c \varphi}$ if $\nu(x)=1$ (see the proof of Proposition 10.5 of [MR]). Here, we are using the same notation for $\varphi$ and the map which $\varphi$ induces on $\bar{H}_{0}$. The next step is to express the integrals $\Phi_{\eta}\left(h_{k}, \mathcal{F}_{\ell}\right), 1 \leq k, \ell \leq 2$, in terms of sums of $\chi_{r}$ over certain $\varphi$ or $c \varphi$-invariant subsets of $\bar{H}_{0}$. This is the analogue of Proposition 10.5 of [MR]. In order to do this, we use Lemma 7.1 to write elements of $\left(\varpi_{E}^{j-1}\left(H_{0} \cap P(r-1)\right)\right)^{\varphi}$ in the form $g \tau \varphi(g)$, where $g \in P(r-1), \tau=1$ if $j=1$, and $\tau \in\left\{\varpi_{L}, \varpi_{E} \delta\right\}$ if $j=2$ (with $\delta$ as in Lemma 7.1). Using these results together with (7.2), (7.3) and Lemma 3.2, and following the proof of Proposition 10.5 of [MR], except with $\mathcal{I}\left(\mathcal{F}_{0}\right), \mathcal{I}\left(\mathcal{F}_{1}\right), H \cap P_{1}$, and $\bar{H}$ of $[\mathrm{MR}]$ replaced by $\Phi_{\eta}\left(h_{1}, \mathcal{F}_{1}\right), \Phi_{\eta}\left(h_{1}, \mathcal{F}_{2}\right)+\Phi_{\eta}\left(h_{2}, \mathcal{F}_{2}\right), H_{0} \cap P_{1}(0)$, and $\bar{H}_{0}$, respectively, results in

Proposition 7.3. Suppose that $f_{E}\left(\theta_{r}\right)=1$. If $f_{0}=2$, assume that $\operatorname{dim} \kappa_{i}=1$ for $1 \leq i \leq r-1$.

(i) $\Phi_{\eta}\left(h_{1}, \mathcal{F}_{1}\right)=\Phi_{\eta}\left(h_{1}, \mathbf{1}_{\left(H_{0} \cap P_{1}(0)\right) h_{1}}\right)\left(\sum_{x \in \bar{H}_{0}^{\varphi}} \chi_{r}(x)\right)$.

(ii) If $e_{0}=1$, then $\Phi_{\eta}\left(h_{1}, \mathcal{F}_{2}\right)=0$ and

$$
\Phi_{\eta}\left(h_{2}, \mathcal{F}_{2}\right)=\theta\left(\varpi_{L}\right) \Phi_{\eta}\left(h_{2}, \mathbf{1}_{\left(H_{0} \cap P_{1}(0)\right) h_{2}}\right)\left(\sum_{x \in \bar{H}_{0}^{\varphi}} \chi_{r}(x)\right) .
$$

(iii) If $e_{0}=2$ and $f\left(L /\left(E_{r-1} \cap L\right)\right)$ is odd, then $\Phi_{\eta},\left(h_{1}, \mathcal{F}_{2}\right)=0$ and

$$
\Phi_{\eta}\left(h_{2}, \mathcal{F}_{2}\right)=\Phi_{\eta}\left(h_{2}, \mathbf{1}_{\left(H_{0} \cap P_{1}(0)\right) h_{2}}\right) \theta\left(\varpi_{L}\right) \theta_{r}(\sqrt{\varepsilon})^{-1}\left(\sum_{x \in \bar{H}_{0}^{-\varphi}} \chi_{r}(x)\right) .
$$

(iv) If $e_{0}=2$ and $f\left(L /\left(E_{r-1} \cap L\right)\right)$ is even, let $\delta$ be as in Lemma 7.1(iii). Then

$$
\begin{aligned}
& \Phi_{\eta}\left(h_{2}, \mathcal{F}_{2}\right)=\Phi_{\eta}\left(h_{2}, \mathbf{1}_{\left(H_{0} \cap P_{1}(0)\right) h_{2}}\right) \theta\left(\varpi_{L}\right) \theta_{r}(\sqrt{\varepsilon})^{-1}\left(\sum_{x \in\left(\mathcal{C}_{\bar{E}} \backslash \mathcal{C}_{\bar{L}}\right) \cap \bar{H}_{0}^{-\varphi}} \chi_{r}(x)\right), \\
& \Phi_{\eta}\left(h_{1}, \mathcal{F}_{2}\right)=\Phi_{\eta}\left(h_{1}, \mathbf{1}_{\left(H_{0} \cap P_{1}(0)\right) \delta \sqrt{\varepsilon}^{-1} h_{2}}\right) \theta\left(\varpi_{E}\right)\left(\sum_{x \in \mathcal{C}_{\bar{L}} \cap \bar{H}_{0}^{-\varphi}} \chi_{r}(x)\right)
\end{aligned}
$$

Remark 7.4. We have used the facts that $\left(H_{0} \cap P_{1}(0)\right) h_{2}=\varpi_{L}\left(H_{0} \cap P_{1}(0)\right) h_{1}$ and that, when $e_{0}=2,\left(H_{0} \cap P_{1}(0)\right) \delta \sqrt{\varepsilon}^{-1} h_{2}=\varpi_{E} \delta\left(H_{0} \cap P_{1}(0)\right) h_{1}$. By arguing along the same lines as in the last part of the proof of Lemma 7.2, we can use Lemma 7.1 to show that if $x \in\left(\varpi_{E}\left(H_{0} \cap P(0)\right)\right)^{\varphi}$ satisfies $\varpi_{E}^{-1} x \in \mathcal{S}_{E-L} \cup \mathcal{S}_{L}$, then $x=g \varphi(g)$ 
for some $g \in G$ if and only if $\varpi_{E}^{-1} x \in \mathcal{S}_{L}$, and that can happen only when $e_{0}=2$ and $f\left(L /\left(E_{r-1} \cap L\right)\right)$ is even. This leads to the conditions on $\Phi_{\eta}\left(h_{1}, \mathcal{F}_{2}\right)$ in parts (ii)-(iv) (see (7.2)).

The signs of the sums appearing in Proposition 7.3 are evaluated as in [MR], using results of $\S 9$ of $[\mathrm{MR}]$, yielding

$$
\begin{gathered}
\Phi_{\eta}\left(h_{1}, \mathcal{F}_{1}\right)>0 \quad \text { and } \quad \Phi_{\eta}\left(h_{1}, \mathcal{F}_{2}\right)=0, \\
(-1)^{f_{0}} \theta\left(\varpi_{L}\right)^{-1} \Phi_{\eta}\left(h_{2}, \mathcal{F}_{2}\right)>0 .
\end{gathered}
$$

Combining this with Lemma 7.2 results in:

Theorem 7.5. Suppose that $f_{E}\left(\theta_{r}\right)=1$. If $f_{0}=2$, assume that $m_{i}=\ell_{i}$ for $1 \leq i \leq r-1$.

(i) If $e_{0}=1$ and $\theta \mid L^{\times} \equiv 1$, then $\Phi_{\eta}\left(h_{k}, f_{\pi}\right)>0, k=1,2$.

(ii) If $e_{0}=2$ and $\theta \mid L^{\times} \not \equiv 1$, then $\Phi_{\eta}\left(h_{k}, f_{\pi}\right)>0, k=1,2$.

\section{MAin RESUlts}

Recall that $E$ is a tamely ramified degree $2 n$ extension of $F, n \geq 2$, and $\theta$ is a unitary character of $E^{\times}$, admissible over the quadratic extension $K$ of $F$, having the property that $\theta \circ \sigma=\theta^{-1}$ for some involution $\sigma$ in $A u t(E / F)$ whose restriction to $K$ is non-trivial. As discussed in $\S 2$ (Lemma 2.1), the supercuspidal representation $\pi$ of $G=G L_{n}(K)$ associated to $\theta$ via Howe's construction $([\mathrm{H}])$ has the property that $\pi \circ \eta \sim \pi$. The fixed field of $\sigma$ is denoted by $L$. Our main results are stated in terms of the values of $\theta$ on $L^{\times}$and certain ramification degrees. We continue to assume that the residue characteristic $p$ of $F$ is odd.

Theorem 8.1. Let $f_{\pi}$ be the finite sum of matrix coefficients of $\pi$ defined in $\S 4$. If $\theta$ satisfies one of the following conditions, then $\Phi_{\eta}\left(h_{k}, f_{\pi}\right)>0, k=1,2$.

(i) $E$ is ramified over $L$ and $\theta \mid L^{\times} \equiv 1$,

(ii) $E$ is unramified over $L$ and

$$
\theta \mid L^{\times}=(-1)^{\operatorname{ord}_{E}(\cdot)(e(K / F)-1) e(E / K),}
$$

with the additional assumption that if $r>1$ and $f_{E}\left(\theta_{r}\right)=1$, then $m_{i}=\ell_{i}, 1 \leq i \leq$ $r-1$.

Remark 8.2. The purpose of the additional assumption in (ii) is to exclude the case where a Heisenberg construction and a representation of a finite general linear group both occur in the inducing data for $\pi$. As remarked in [MR], we expect that the result still holds in that case.

Proof of Theorem 8.1. If (i) holds, the result follows from Proposition 5.3 and Lemma 5.4(ii).

Assume that (ii) holds. If $f_{E}\left(\theta_{r}\right)=1$ and $f_{0}=1$, then $e(K / F)=2$ and $e\left(E_{r-1} / K\right)$ is odd, by Lemma $5.4(\mathrm{i})$. Therefore $e(E / K)=e\left(E_{r-1} / K\right)$ is odd. Note that in this case $m_{i}=\ell_{i}$ is guaranteed by Lemma 5.4(iv). If $f_{E}\left(\theta_{r}\right)=1, f_{0}=2$, and $e(K / F)=2$, then, by Lemma 6.6(i), the assumption $m_{i}=\ell_{i}, 1 \leq i \leq r-1$, implies that $e(E / K)$ is even. We conclude that in the case $f_{E}\left(\theta_{r}\right)=1$, Theorem 7.5 coincides with this theorem. 
For the remainder of the proof, suppose that (ii) holds and $f_{E}\left(\theta_{r}\right)>1$. Let $\mu$ be as defined in $\S 5$. It follows from Lemma 5.2 that, given $1 \leq i \leq r$ and $x \in H_{0}^{\varphi}$,

$$
\text { If } m_{i}=\ell_{i}, \text { then } \begin{gathered}
\chi_{i}(x)=\kappa_{i}(x)=\theta_{i}\left(N_{E / E_{i}}(\mu(x))\right), \\
\Lambda\left(\operatorname{det}_{0}(x)\right)=\Lambda\left(N_{E / K}(\mu(x))\right) .
\end{gathered}
$$

Next, suppose that $m_{i} \neq \ell_{i}$ for some $i$. Let $H_{i}$ and $H_{i}^{\prime}$ be as in $\S 6$. By Lemma 3.2(i), given $x \in H_{0}^{\varphi}$, there exist $y \in\left(E^{\times} H_{i}\right)^{\varphi}=\left(E^{\times} \mathcal{K}_{i-1}\right)^{\varphi}$ and $z \in \mathcal{L}_{i-1}$ such that $x=y z$. By definition of $\kappa_{i}$ (see the beginning of $\S 5$ ),

$$
\chi_{i}(x)=\chi_{i}(y) \psi\left(\operatorname{tr}\left(c_{i}(z-1)\right)\right) .
$$

Note that $z-1 \in \mathcal{B}_{\ell_{i-1}}(0)$, so that

$$
\begin{array}{r}
y^{\prime}(z-1) y^{\prime-1} \in(z-1)+\mathcal{B}_{\ell_{i}+\ell_{i-1}}(0) \subset(z-1)+\mathcal{B}_{f_{E}\left(\theta_{i} \circ N_{E / E_{i}}\right)}(0), \\
\text { if } y^{\prime} \in P_{\ell_{i}}(0) .
\end{array}
$$

Now $y \in E^{\times} \mathcal{K}_{i-1}=E^{\times} \mathcal{K}_{i} P_{\ell_{i}}(i-1)$, and $c_{i}$ commutes with $E^{\times} \mathcal{K}_{i}$, so

$$
\operatorname{tr}\left(c_{i}\left(y(z-1) y^{-1}\right)\right)=\operatorname{tr}\left(c_{i}(z-1)\right) .
$$

By definition of $\varphi, \operatorname{tr}(\varphi(X))=\sigma(\operatorname{tr}(X)), X \in \mathfrak{g}$. As $x$ and $y$ are $\varphi$-invariant, it follows that $\varphi(z)=y z y^{-1}$. Thus, using Lemma 2.3(iii), we find

$$
\sigma\left(\operatorname{tr}\left(c_{i}(z-1)\right)\right)=-\operatorname{tr}\left(c_{i}\left(y(z-1) y^{-1}\right)\right)=-\operatorname{tr}\left(c_{i}(z-1)\right) .
$$

Combining this with $\psi=\psi_{0} \circ \operatorname{tr}_{K / F}($ see $\S 2)$, results in $\psi\left(\operatorname{tr}\left(c_{i}(z-1)\right)\right)=1$. Thus $\chi_{i}(x)=\chi_{i}(y)$. As $y \in E^{\times} H_{i}$, we may apply results of $\S 6$ to evaluate $\chi_{i}(y)$. Let $\nu$ be as in $\S 3$. Note that $\nu(x)=\nu(y)$ and $\mu(x)=\mu(y)$. If $y$ is conjugate to an element of $E^{\times} H_{i}^{\prime}$, then by Lemma 6.3 and Corollary 6.5,

$\chi_{i}(x)$ is a positive multiple of

$$
\begin{cases}(-1)^{\nu(x)} \theta_{i}\left(N_{E / E_{i}}(\mu(x))\right), & \text { if } e(E / K) \text { is odd, } e\left(E_{i-1} /\left(E_{i-1} \cap L\right)\right)=2, \\ & \text { and } e\left(E_{i} /\left(E_{i} \cap L\right)\right)=1, \\ \theta_{i}\left(N_{E / E_{i}}(\mu(x))\right), & \text { otherwise. }\end{cases}
$$

As shown in Lemma 6.6, the first case in (8.2) can occur if and only if $e(E / K)$ is odd and $e(K / F)=2$, and then it must occur for exactly one $i, 1 \leq i \leq r$.

It follows from (8.1), (8.2), Lemma 6.6, and the definition of $\kappa$ (see $\S 5$ ), that if $x \in H_{0}^{\varphi}$ and $\chi_{\kappa}(x) \neq 0$, then $\chi_{\kappa}(x)$ is a positive multiple of

$$
\theta(\mu(x))(-1)^{\nu(x)(e(K / F)-1) e(E / K)} .
$$

In particular, if $x \in\left(E^{\times} P_{m_{r}}(r-1) \cdots P_{m_{1}}(0)\right)^{\varphi}$, and $\theta$ is as in (ii), then $\chi_{\kappa}(x)>0$. Thus, by (4.2), $\Phi_{\eta}\left(h_{k}, f_{\pi}\right)>0, k=1,2$.

As in $\S 4$, we let $G^{\prime}$ and $G^{\prime \prime}$ be the $F$-rational points of the quasi-split unitary groups in $2 n$ and $2 n+1$ variables, respectively, defined with respect to $K / F$. Recall that $P^{\prime}$ and $P^{\prime \prime}$ are parabolic subgroups of $G^{\prime}$ and $G^{\prime \prime}$, respectively, having Levi components isomorphic to $G$ and $G \times K^{1}$, respectively. Given a character $\xi$ of $K^{1}$, the supercuspidal representation $\Pi_{\xi}$ of $G \times K^{1}$ is defined by $\Pi_{\xi}(x, \alpha)=$ $\pi(x) \xi\left(\operatorname{det}_{0}(x \eta(x)) \alpha\right), x \in G, \alpha \in K^{1}$. We can combine Theorem 8.1 and Goldberg's reducibility criterion (Theorem 4.1) to obtain results concerning reducibility of the representations $I(\pi)=\operatorname{Ind}_{P^{\prime}}^{G^{\prime}}(\pi \otimes 1)$, and $I\left(\Pi_{\xi}\right)=\operatorname{Ind}_{P^{\prime \prime}}^{G^{\prime \prime}}\left(\Pi_{\xi} \otimes 1\right)$. 
Theorem 8.3. Suppose that the admissible character $\theta$ satisfies (i) or (ii) of Theorem 8.1. Then $I(\pi)$ is irreducible and $I\left(\Pi_{\xi}\right)$ is reducible (for any $\xi$ ).

It is likely that the above conditions on $\theta$ are necessary and sufficient for irreducibility of $I(\pi)$ (equivalently, for reducibility of $I\left(\Pi_{\xi}\right)$ ). See $\S 11$ of $[\mathrm{MR}]$ for a discussion of the analogous situation for induced representations of split classical groups. In order to show sufficiency, it would be necessary to prove that $\Phi_{\eta}\left(h_{1}, f\right)=-\Phi_{\eta}\left(h_{2}, f\right)$ for all choices of matrix coefficients $f$ of $\pi$.

Conjecture 8.4. $I(\pi)$ is irreducible if and only if $\theta \mid L^{\times}$satisfies

$$
\theta \mid L^{\times}= \begin{cases}1, & \text { if } f(E / L)=1, \\ (-1)^{\operatorname{ord}_{E}(\cdot)(e(K / F)-1) e(E / K)}, & \text { if } f(E / L)=2 .\end{cases}
$$

Combining Theorem 8.3 and a result of Goldberg, we can get information about reducibility of representations induced from non-unitary supercuspidal representations of $G$ and of $G \times K^{1}$. Let $|\cdot|_{K}$ denote the normalized valuation on $K$. For $s$ a non-negative real number, set

$$
I(s, \pi)=I\left(\pi \otimes\left|\operatorname{det}_{0}(\cdot)\right|_{K}^{s / 2}\right)
$$

and

$$
I\left(s, \Pi_{\xi}\right)=I\left(\Pi_{\xi} \otimes\left|\operatorname{det}_{0}(\cdot)\right|_{K}^{s}\right) .
$$

Corollary 8.5. Suppose that the admissible character $\theta$ satisfies (i) or (ii) of Theorem 8.1. Then $I(s, \pi)$ is reducible if and only if $s=1$, and $I\left(s, \Pi_{\xi}\right)$ is irreducible for all $s>0$.

Proof. By Theorem 8.3, $I(\pi)=I(0, \pi)$ is irreducible, and $I\left(\Pi_{\xi}\right)=I\left(0, \Pi_{\xi}\right)$ is reducible. The result then follows from Theorems 3.1 and 6.3 of [G2].

\section{REFERENCES}

A. J. D. Adler, Self-contragredient supercuspidal representations of $G L_{n}$, Proc. Amer. Math. Soc. 125 (1997), no. 8, 2471-2479. MR 97j:22038

G1. D. Goldberg, Reducibility of generalized principal series representations for $U(2,2)$, Comp. Math. 86 (1993), 245-264. MR 94i:22039

G2. Some results on reducibility for unitary groups and local Asai L-functions, J. Reine Angew. Math. 448 (1994), 65-95. MR 95g:22031

H. R. Howe, Tamely ramified supercuspidal representations of $G L_{n}$, Pacific J. Math. 73 (1977), 437-460. MR 58:11241

K1. D. Keys, Principal series representations of special unitary groups over local fields, Comp. Math. 51 (1984), 115-130. MR 85d:22031

K2. $\longrightarrow, L$-indistinguishability and R-groups for quasi-split groups: unitary groups in even dimension, Ann. scient. Ec. Norm. Sup. t.20 (1987), 31-64. MR 88m:22042

KS1. R. Kottwitz and D. Shelstad, Twisted endoscopy I: definitions, norm mappings and transfer factors, preprint.

KS2. _ Twisted endoscopy II: basic global theory, preprint.

M. A. Moy, Local constants and the tame Langlands correspondence, Amer. J. Math. 108 (1986), 863-930. MR 88b:11081

MR. F. Murnaghan and J. Repka, Reducibility of some induced representations of split classical p-adic groups, Compositio Math., to appear. 
R. J.D. Rogawski, Automorphic representations of unitary groups in three variables, Ann. Math. Stud. 123, Princeton, NJ, 1990. MR 91k:22037

Sh. F. Shahidi, Twisted endoscopy and reducibility of induced representations for p-adic groups, Duke J. Math. 66 (1992), 1-41. MR 93b:22034

Department of Mathematics, University of Toronto, 100 St. George Street, ToRonto, Canada, M5S 3G3

E-mail address: fiona@math.toronto.edu

Department of Mathematics, University of Toronto, 100 St. George Street, ToRONTO, CANADA, M5S 3G3

E-mail address: repka@math.toronto.edu 